\title{
Lipid lowering: education is key
}

$\mathrm{E}$ ducating the general population on the safe and best use of lipid-lowering agents is imperative, given the widespread availability of such therapies. Whether or not you are in favor of the ongoing argument for the availability of statins without prescription, the reality is that nonprescription statins are already available in the UK and are likely to become available in other countries at some point in the near future. Furthermore, nonpharmaceutical supplements that act to decrease LDL-cholesterol levels are easily accessible worldwide. The current debate, therefore, should not center on whether we should allow nonprescription statins, but instead focus on how we should educate people on the use of lipid-lowering therapies.

Evidence is mounting for the additional benefit of statins over recommended lifestyle changes in almost every patient group. Indeed, as recently as June this year, researchers who undertook a meta-analysis of 10 randomized trials concluded that statins effectively reduce cardiovascular risk in individuals without established cardiovascular disease but with cardiovascular risk factors, such as diabetes, hypercholesterolemia, and hypertension (Brugts, J. J. et al. BMJ 338, b2376 [2009]). Furthermore, many individuals who are candidates for lipid-lowering therapy are not undergoing treatment, even though they are aware of their hypercholesterolemia, or have risk factors that are inadequately controlled (Pletcher, M. J. et al. Ann. Int. Med. 150, 243-254 [2009]; Gotto, A. Circulation 120, 3-5 [2009]). In response to both the growing evidence for the benefits of statin therapy and the current 'treatment gap', and because they think it would enhance adherence, more and more clinicians, pharmaceutical companies, and regulatory agencies around the world are arguing for the implementation of nonprescription statins.

The major arguments against nonprescription statins are that individuals in the general population cannot self-diagnose hypercholesterolemia, and that those with severe hypercholesterolemia require aggressive and monitored statin treatment. Both diagnosis and monitoring of hypercholesterolemia require the assessment of plasma lipid levels by a medical professional. Furthermore, some clinicians argue that there comes a point when the risk of adverse effects outweighs the potential benefits of statins in individuals who are at very low cardiovascular risk. These points are important to consider when deciding how best to deal with easily available lipid-lowering therapy.

In mid 2004, statins became available in the UK without the need for a prescription. Instead of implementing a straight 'over-the-counter' system, however, the UK health authorities licensed simvastatin $(10 \mathrm{mg})$ to be provided from 'behind the counter' to patients judged by the pharmacist as being at moderate risk of developing coronary artery disease, based on certain criteria. This system nullifies many of the concerns related to overthe-counter availability, firstly, by allowing pharmacists to determine whether an individual is at moderate risk of coronary artery disease and thus whether the benefits of statin therapy would outweigh any potential adverse effects. Secondly, this system would potentially allow pharmacists to identify patients who are at high risk and should be receiving aggressive medical treatment and monitoring by a doctor. Furthermore, and very importantly, this system gives pharmacists the opportunity to provide individuals with information about the risks and therapeutic requirements associated with lipid-lowering therapy. Indeed, pharmacists could potentially advise patients to undergo follow-up assessments of their cholesterol levels at appropriate intervals. Finally, although more regulated than an over-the-counter system, a behind-the-counter system could be predicted to result in the same high level of patient adherence to statin therapy that is expected from the former system, owing to its comparable accessibility. The issue of adherence is important; in addition to the demonstrated benefits of statins, a study of 141,086 Canadian patients who initiated statins for primary prevention demonstrated that adherent patients (defined as those dispensed a supply of more than 120 days of statin medication in the 1 -year baseline period) were more likely to use health-screening services than less adherent patients (Dormuth, C. R. et al. Circulation 119, 2051-2057 [2009]).

The FDA has rejected multiple applications over the last 10 years for statins to be made available without prescription. At present in the US, pharmaceuticals are only available by prescription or over the counter. Perhaps the US and other countries around the world should learn from the UK's experiences and consider training pharmacists in the implementation of behindthe-counter systems and in the educating and monitoring of individuals on lipid-lowering therapy.

If and when nonprescription statins become available outside the UK, it will be crucial for patients to be well informed about lipid-lowering therapies before they go to the pharmacy. One way to ensure this knowledge would be to implement a system where patients have to submit a form-with information on their risk factor profile, their contraindications to statin therapy, the potential for adverse effects, and the contact details of a
4 ...there

is a clear need for us to focus ... on educating the general public on ... lipid-lowering therapy 77

Valentin Fuster is the Editor-in-Chief and Bryony M. Mearns is the Editor of Nature Reviews Cardiology.

Competing interests V. Fuster declares an association with BG Medicine Inc.

See the article online for full details of the relationship.

B. M. Mearns declares no competing interests. 
health-care professional for follow-up - to the pharmacist at the point-of-sale. This strategy would ensure that patients have had appropriate screening and are reasonably well-informed about the safe and best use of statins before they buy these drugs. This approach would also allow the pharmacist to double-check the patient's suitability for statin therapy. A strategy of this type would be most effective if coupled with implementation of a campaign for widespread education on lipid-lowering therapies in the general population.

Whether or not a behind-the-counter system is put in place, all nonprescription statins would of course need to be clearly labeled so that the patient could refer to this information when not in the presence of a medical professional, as reiterated by the FDA on numerous occasions. Studies evaluating whether clear labeling can be effective in discouraging individuals who are not appropriate candidates for statin therapy have provided mixed results and more research on how to improve the labeling is required. Once optimized for this purpose, however, labels should also be used to educate and remind individuals of the need for regular lipid assessment and the importance of concurrent lifestyle changes. When statins were used for primary prevention in individuals aged 45-80 years in the Indian polypill study, the rate of discontinuation owing to adverse effects of statins was less than $4 \%$, which was similar to the rate observed for the other polypill components studied (The Indian Polycap Study [TIPS]. Lancet 373, 1341-1351 [2009]). Nevertheless, labels on any nonprescription statins should of course also remind the individuals of the potential adverse effects associated with statin use.

In addition to a regulated, pharmaceutical therapy for lipid lowering (that is, statins), many supplements thought to have lipid-lowering effects are available to the general population worldwide. Indeed, a small randomized controlled trial published in June this year demonstrated that one of these supplements, red-yeast rice, was effective in lowering LDL-cholesterol levels in statinintolerant patients (Becker, D. J. et al. Ann. Intern. Med. 150, 830-839 [2009]). As more of these supplementswhich are not regulated by health authorities such as the FDA-are shown to be effective in randomized controlled trials, the need for far-reaching education campaigns on lipid lowering will become increasingly important. This education should not be driven by pharmaceutical or 'nutraceutical' agencies wanting (or at least perceived to be wanting) to push their products but, rather, by health authorities who can provide consumers with trusted advice. Whether or not statins themselves are made available without a prescription, education campaigns should be implemented and focus on the importance of regular cholesterol assessments, following dosing recommendations made by medical professionals, and making concurrent beneficial lifestyle changes.

Given that the cost of nonprescription medicine is the responsibility of the consumer, only individuals who perceive that they will be benefit from lipid-lowering medication are likely to be interested in taking this therapy. Nevertheless, education campaigns and semi-regulated systems for pharmaceuticals, such as the behind-thecounter system used in the UK, would reduce the likelihood of inappropriate use whilst also potentially increasing adherence to therapy in those who would benefit from it.

In conclusion, irrespective of whether statins are made available without a prescription outside the UK, there is a clear need for us to focus our attention on educating the general public on the risks and therapeutic requirements associated with lipid-lowering therapy. This education should not only be provided by pharmacists at point-ofsale, but also through clear labeling on packaging and via widespread health-authority-driven education and advertising campaigns. Shifting our focus to education on lipid lowering will save lives.

doi:10.1038/nrcardio.2009.164 